\title{
Correction: Respiratory viral pathogens among Singapore military servicemen 2009 - 2012: epidemiology and clinical characteristics
}

Xin Quan Tan ${ }^{1,2+}$, Xiahong Zhao ${ }^{3+}$, Vernon J Lee ${ }^{1,3^{*}}$, Jin Phang Loh ${ }^{4}$, Boon Huan Tan ${ }^{4}$, Wee Hong Victor Koh ${ }^{4}$, Sock Hoon $\mathrm{Ng}^{4}$, Mark I-Cheng Chen ${ }^{3,5}$ and Alex Richard Cook $3,6,7,8$

\section{Text}

A minor error was found in Table 1 that described and compared the demographics amongst all participants, cases, controls and mono-infections after publishing this work [1]. For recruits, the number and the corresponding proportion in each group was under-reported. This was caused by discrepancies in the database in how recruits were coded that were not noticed until now. We have recoded recruits in the database and have re-analysed data. The differences in proportions of recruits amongst all groups remained significant with the revised data (Table 1). These errors only affect this table. All the other results and conclusions remain.

We apologise for this oversight, and the inconvenience that we might have brought.

Table 1 Demographics of all participants, cases, controls and mono-infection cases

\begin{tabular}{|c|c|c|c|c|c|}
\hline Characteristics & All (Count,\%) & Cases (Count,\%) & Controls (Count,\%) & Mono-infection (Count,\%) & p-value \\
\hline Age (Mean, SD) & $20.8(3.1)$ & $20.7(3.1)$ & $21.1(2.9)$ & $20.7(3.2)$ & 0.781 \\
\hline Male & 9055 (99.8) & $7713(99.7)$ & $1342(99.9)$ & $3422(99.8)$ & 0.902 \\
\hline Current Smoker & $2475(27.3)$ & $2090(27.0)$ & $385(28.6)$ & $887(25.9)$ & 0.220 \\
\hline Recruit & $6048(66.6)$ & $5568(72.0)$ & $480(35.7)$ & $2495(72.7)$ & $<0.001$ \\
\hline Asthma & $1833(20.2)$ & $1597(20.7)$ & $236(17.6)$ & $721(21.0)$ & 0.051 \\
\hline Heart Disease & $102(1.1)$ & $83(1.1)$ & $19(1.4)$ & $37(1.1)$ & 0.748 \\
\hline Total & $9077(100.0)$ & $7733(100.0)$ & $1344(100.0)$ & $3430(100.0)$ & \\
\hline
\end{tabular}

† By ANOVA test, comparing mean age, and by Pearson's chi-square test, comparing proportions across all categories.

\footnotetext{
* Correspondence: vernonljm@hotmail.com

${ }^{\dagger}$ Equal contributors

'Biodefence Centre, Ministry of Defence, Singapore, Singapore

${ }^{3}$ Saw Swee Hock School of Public Health, National University of Singapore,

Singapore, Singapore

Full list of author information is available at the end of the article
} 


\section{Author details}

${ }^{1}$ Biodefence Centre, Ministry of Defence, Singapore, Singapore. ${ }^{2}$ Preventive Medicine Residency Programme, National University Health System, Singapore, Singapore. ${ }^{3}$ Saw Swee Hock School of Public Health, National University of Singapore, Singapore, Singapore. ${ }^{4}$ Defence Medical and Environmental Research Institute, Singapore, Singapore. ${ }^{5}$ Department of Clinical Epidemiology, Tan Tock Seng Hospital, Singapore, Singapore. ${ }^{6}$ Yale-NUS College, National University of Singapore, Singapore, Singapore.

${ }^{7}$ Program in Health Services and Systems Research, Duke-NUS Graduate Medical School, Singapore, Singapore. ${ }^{8}$ Department of Statistics and Applied Probability, National University of Singapore, Singapore, Singapore.

Received: 11 June 2014 Accepted: 12 June 2014

Published: 24 July 2014

\section{Reference}

1. Tan XQ, Zhao X, Lee VJ, Loh JP, Tan BH, Koh WH, Ng SH, Chen Ml, Cook AR: Respiratory viral pathogens among Singapore military servicemen 2009-2012: epidemiology and clinical characteristics. BMC Infect Dis 2014, 14(1):204.

doi:10.1186/1471-2334-14-326

Cite this article as: Tan et al:: Correction: Respiratory viral pathogens among Singapore military servicemen 2009 - 2012: epidemiology and clinical characteristics. BMC Infectious Diseases 2014 14:326.

\section{Submit your next manuscript to BioMed Central and take full advantage of:}

- Convenient online submission

- Thorough peer review

- No space constraints or color figure charges

- Immediate publication on acceptance

- Inclusion in PubMed, CAS, Scopus and Google Scholar

- Research which is freely available for redistribution 